\title{
Case Report - Mortality Due to Underestimation of Soft Tissue Injury and Misdiagnosis of Rhabdomyolysis
}

\author{
Tan $L J^{a}$, Tan $R Z^{a}$, Shafie $M S^{a}$, Mohd Nor $F^{a}$, Shamsuddin $H^{2}$, Md Onzer $M A^{3}$ \\ $a^{*}$ Department of Pathology, Universiti Kebangsaan Malaysia Medical Centre, \\ Jalan Yaacob Latif, Bandar Tun Razak, Cheras, 56000 Kuala Lumpur, Malaysia. \\ ' Ibu Pejabat Polis Daerah Johor Bahru Utara

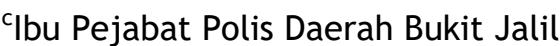

\section{ABSTRACT}

Introduction Rhabdomyolysis is characterized by the breakdown of muscle tissue, and release of intracellular muscle constituents into the blood circulation. The severity of the illness may range from asymptomatic elevation in serum muscle enzymes to a life-threatening disease. One disease may behave differently in different way in different individuals. The first patient was involved in a motor vehicle accident and developed acute kidney injury on the third day of admission. The second patient complained of lower limb weakness, and was discharged with vitamin D supplements. Both patients' conditions were not properly diagnosed or treated, and succumbed to death. Autopsies were conducted in both cases, in which rhabdomyolysis were diagnosed. This case report emphasizes the importance of making a correct diagnosis of rhabdomyolysis Progression of the disease and its complications should be monitored closely. Timely management may save life in high risk patients.

KEYWORDS: Soft tissue injury, rhabdomyolysis, forensic.

\section{INTRODUCTION}

Soft tissue injury constitutes damage to muscle, ligament and tendon, which is the commonest injury seen in trauma cases. It usually resolves by itself without long term sequelae. However, most of the cases were treated lightly, and patients were not investigated or monitored for potential complications. ${ }^{1}$ In reality, soft tissue injury can eventually contribute to death either immediately or maybe delayed for some days or months. The causes of immediate death upon soft tissue injury can be due to rapid, extensive hemorrhage into subcutaneous tissue, severe pain, shock, reflex vagal inhibition or mechanical injury to vital organs. For instance, in commotio cordis, a blow to the chest in the precordial region may disrupt the heart rhythm, and contribute to death without even showing any

Corresponding Author

Prof. Dr. Faridah Mohd Nor

Forensic Unit, Department of Pathology

Universiti Kebangsaan Malaysia Medical Centre Jalan Yaacob Latif, Bandar Tun Razak, Cheras 56000 Kuala Lumpur, Malaysia

Tel. No : $+603-91455368$

Email: mnfaridah@gmail.com significant haemorrhage in the internal organ. The causes of delay complication upon soft tissue injury may include infection, gangrene or necrosis, crush syndrome, fat embolism, air embolism, thrombosis and supervening diseases from traumatic lesion. ${ }^{2}$

We, herein report two cases of hospitalized patients with diagnoses of soft tissue injury following motor vehicle collision and physical assault, respectively. Patients had succumbed to death after few days of injury. The deceased patients were brought to the mortuary by the police, and rhabdomyolysis were diagnosed from autopsy in both cases. The aim of this case report is to emphasize the importance of making an early recognition of rhabdomyolysis, and to provide timely management of high risk cases in particular.

\section{CASE REPORT 1}

A 48-year-old gentleman with no underlying illness, had skidded while riding a motorcycle, and was brought to the hospital. He presented with a Glasgow Coma Scale (GCS) of 14/15 (M5V5E4), blood pressure of $130 / 75$, heart rate was 85 per minute and respiratory rate was 18 per minute. He showed 
multiple abrasions with swelling of the right thigh. Laboratory investigations showed normal full blood count and slightly elevated serum urea $9 \mathrm{mmol} / \mathrm{L}$ (reference: 3.2- 7.3) and normal creatinine 97.2 $\mu \mathrm{mol} / \mathrm{L}$ (reference: 63.6- 110.5), potassium 5.0 mmol/L (reference: $3.5-5.1$ ) and sodium $139 \mathrm{mmol} /$ L (reference: 135- 145). He was admitted to the neurosurgical department with a diagnosis of cerebral concussion in view of a short episode of loss of consciousness after injury with normal findings in brain CT scan.

During the first three days of admission, his GCS level remained between 13 and 14, and no further laboratory investigations were done or repeated. The doctor referred the patient to a medical team for a sleep study in view of his loud snoring and high blood glucose level ranging from 9 to 14 $\mathrm{mmol} / \mathrm{L}$. Subsequently, he became unresponsive and had cardio-respiratory arrest. Cardio-pulmonary resuscitation was done, he was intubated and ventilated. Infusion of inotrope, was commenced and was then transferred to a neurosurgical intensive unit (ICU). Patient was declared death after four hours of ICU admission. Upon receiving the body at the mortuary, the cause of death was given as 'acute kidney injuries with unknown cause'. The police requested for postmortem to determine the cause of death.

An autopsy was performed, and external examination showed multiple healed abrasions on extremities with soft tissue swelling of the right thigh. Internal examination showed two foci of subarachnoid hemorrhages $3 \times 3 \mathrm{~cm}$ and $2 \times 2 \mathrm{~cm}$ on the right parietal and right frontal lobes, respectively. The descending colon showed some ischaemic changes. Chemical analysis of urine showed presence of myoglobin.

Histology of brain showed small foci of subarachnoid hemorrhages. Lung sections showed diffuse alveoli damage with fibrin deposition within the capillaries. Sporadic fat embolus was seen inside the pulmonary vessels with surrounding congestion. Sections of both kidneys showed streak-like casts inside the tubular lumen, cellular infiltration of the renal tubular wall with positive stains for myoglobin (Fig. la, Ib). Sections of heart showed generalized hypertrophic changes with subendocardial fibrosis of both ventricles and sporadic interstitial fibrosis of myocardium.

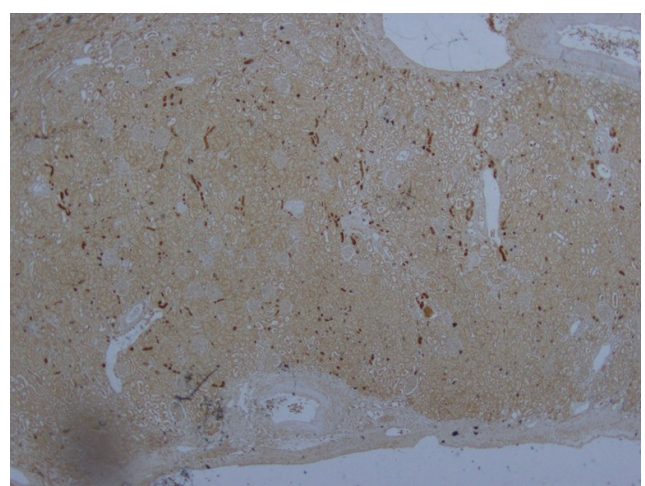

Fig. la Low power view of immunohistochemical staining of kidney tissue with myoglobin. (x400 magnification)

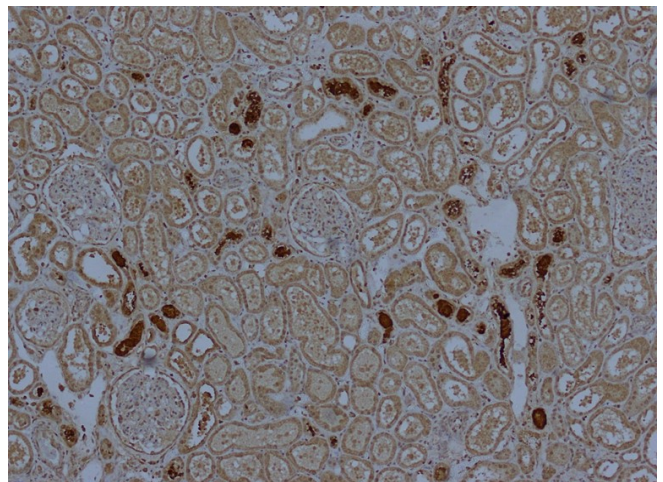

Fig. Ib High power view of immunohistochemical staining of kidney tissue with myoglobin. (x100 magnification)

\section{CASE REPORT 2}

A 50-year-old foreign citizen had come to reside in Malaysia with no legal documentations. He had no underlying illness, and was detained by the immigration officer for few months in a 12-person sharing cell. In previous six months while he was in detention, he had pain and weakness in both legs, and was unable to walk. He received treatment from the nearest clinic few times, and was treated conservatively with vitamins and analgesics to ease the pain. At the time, he was diagnosed with vitamin $D$ deficiency. Few days later, he was admitted to a hospital for one week. He was diagnosed with resolved electrolyte imbalance with soft tissue injury. He was discharged, and three days later, he was found dead in his cell.

Postmortem examination revealed few scars scattered on anterior aspects of both lower legs. Sectioning of the skin showed multiple extensive subcutaneous haematoma of differing ages on both legs and ventral surface of right foot. Both right and left calf muscles were contused with significant amount of blood accumulated within the fasciae (Fig. II, III). Chemical analysis showed elevated total 
creatine kinase level of $14085 \mathrm{U} / \mathrm{L}$ (reference: 30 200), and myoglobin was detected in urine. Histology of kidneys showed accumulation of myoglobin casts within the lumen and cytoplasm of the renal tubules. Section of calf muscle showed extravasation of erythrocytes in interstitium and muscle fibres, hemosiderin pigment in macrophages with necrotic muscle and neutrophilic and/ or lymphocytic infiltrations.

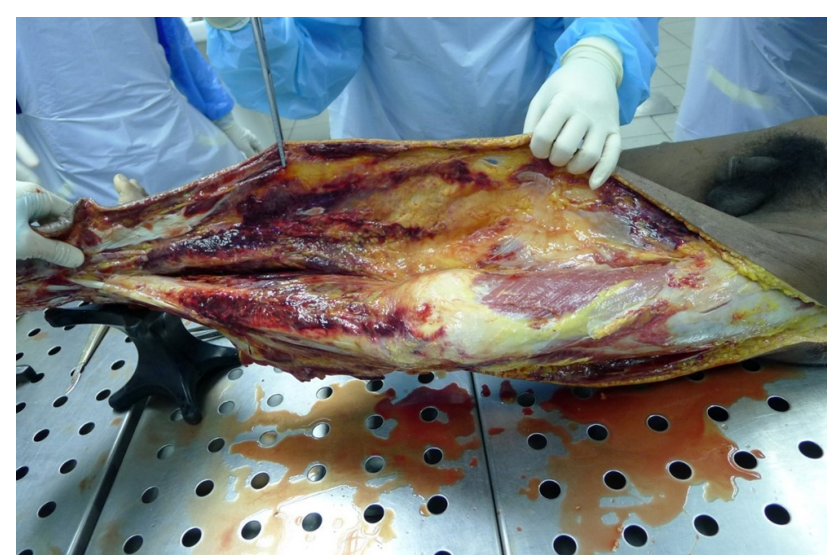

Fig. II Extensive subcutaneous and muscle contusion of left lower limb.

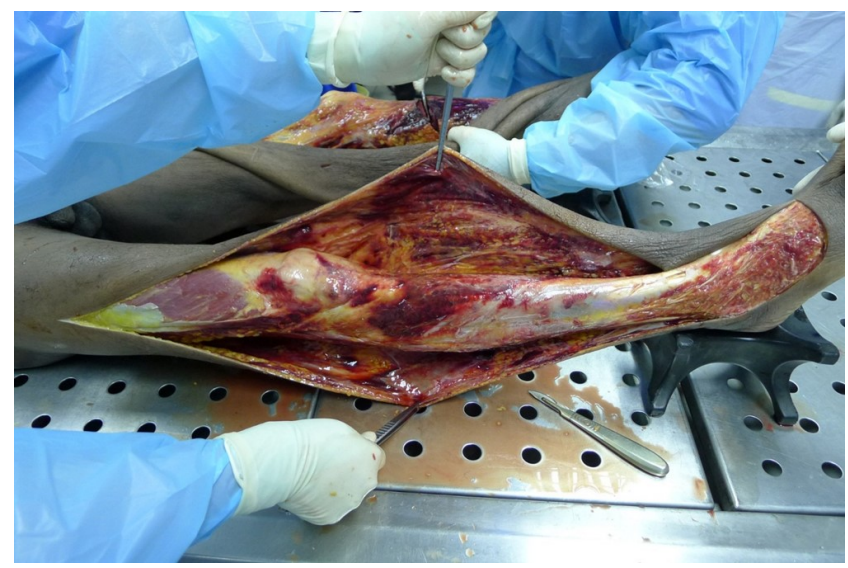

Fig. III Extensive subcutaneous and muscle contusion of right lower limb.

\section{DISCUSSION}

Rhabdomyolysis is characterized by breakdown of muscle tissue and release of intracellular muscle constituents into the blood circulation. The severity of illness ranges from asymptomatic elevation in serum muscle enzymes through to a life-threatening disease associated with extreme enzyme elevation, electrolyte imbalance and acute kidney injury. ${ }^{3}$ Excessive myoglobin may circulate into the kidney, thus causing renal tubular obstruction, impaired kidney filtration function and acute kidney injury. When kidney is unable to remove toxins from the blood circulation, accumulation of toxin may cause multiple organ failure, and finally results in death.
Electrolyte imbalance such as hyperkalemia and hypocalcemia may result in arrhythmias. Release of tissue factor into the circulation may result in disseminated intravascular coagulation and internal hemorrhagic complications.

Causes of rhabdomyolysis may be classified into four main types: ${ }^{3}$

1) Traumatic or muscle compression (e.g. crush syndrome or prolonged immobilization)

2) Non-traumatic exertional conditions (e.g. marked exertion in untrained individuals, hyperthermia or metabolic myopathies)

3) Non-traumatic non-exertional conditions (e.g. drug or toxin, infection or electrolyte disorders)

4) Miscellaneous (e.g. status asthmaticus, capillary leak syndrome)

Clinically, patient may present with muscle pain, swelling and dark colored urine due to the presence of myoglobinuria. The hallmark of laboratory investigation is elevation of serum total creatine kinase (preferable CKMM- specific for skeletal muscle) and positive urine myoglobin. ${ }^{4}$ The damaged muscle tissue will release intracellular electrolytes into the blood circulation, and causes hyperkalaemia, hyperphosphatemia, and hypocalcaemia due to deposition of calcium salts in damaged muscles. Acute kidney injury associated with rhabdomyolysis is the most serious complication, and may be life-threatening. ${ }^{5}$ The exact mechanism of renal impairment in rhabdomyolysis is unclear, but experimental evidence suggests that intrarenal vasoconstriction, direct and ischaemic tubular injury with tubular obstruction, all played some role in the pathological mechanism of the disease. ${ }^{6}$ Other investigations, which should be taken into consideration include ECG monitoring of cardiac arrhythmia in cases of hyperkalemia or hypocalcemia, levels of muscle enzymes such as lactate dehydrogenase, aldolase, carbonic anhydrase III and aminotransferase, troponin- $T$ and coagulation profile in cases of disseminated intravascular coagulopathy. ${ }^{7}$

Tubular obstruction occurs mainly in the distal tubules, but direct tubular cytotoxicity occurs commonly in the proximal tubules. Histology of muscle may show extensive muscle necrosis with hemorrhages and minimal inflammatory cells infiltrations. Histology of kidney may show renal 
tubules with coarsely granular proteinaceous deposits found either in clumped or forming a ring within the lumina of the tubules. Immunohistochemical stain for myoglobin may show a strong positivity within granular tubular casts and cytoplasm of tubular cells. $^{8}$

The general approach for a patient with rhabdomyolysis is to identify and manage the cause, correct the hypovolaemia and electrolyte disturbance such as hyperkalaemia, hypocalcaemia, acidosis, and proceed with dialysis for renal impairment, where indicated. ${ }^{9}$ The overall mortality rate of a patient with rhabdomyolysis is approximately $5 \%$, which depends on the aetiology and underlying co-morbidity conditions of the patient.10 A good prognosis may be expected, where treatment is instituted early and promptly. ${ }^{9,10}$

In the first case, the right thigh swelling was not identified despite visible abrasions seen on the body. The elevated urea level seen during admission was not taken seriously. Besides, daily urea and creatinine monitoring and other electrolytes for possibility of rhabdomyolysis were not requested. Histology of kidney showed changes of renal damage secondary to rhabdomyolysis due to soft tissue injury. Complication of rhabdomyolysis such as disseminated intravascular coagulopathy may result in fibrin deposition in capillaries of alveoli, ischaemic changes of bowel and diffuse alveolar damage with mechanical lung injury. The cause of death was rhabdomyolysis and diffuse alveolar damage due to soft tissue injury. It was worth mentioning that low GCS level was due to traumatic subarachnoid haemorrhages, which were not detected by CT scan of brain.

In the second case, the deceased was tortured and physically abused. He received blows during his detention in the immigration office. However, the inhumane physical abuse was not identified early despite multiple visits to medical centres. The history obtained by the doctor was incomplete for several reasons. He, being a foreigner may have some communication problem to relate the incident himself to the doctor. Besides, the abusing incident that happened in prison may not be revealed by the patient for reasons of fear. In comparison with the first case, the second patient developed complication of soft tissue injury during his first hospital presentation. Electrolyte imbalance was merely caused by poor nutrition intake and longterm, lack of sunlight exposure in prison. Thus, the condition was not investigated accordingly. He was discharged with oral supplement and analgesics.

In some cases of rhabdomyolysis, electrolyte imbalance can be temporary resolved with good hydration and rest. However, in this case, the etiology was not promptly identified and treated. The patient's condition had rapidly deteriorated and died after three days being discharged from the hospital. In both cases, soft tissue injury was underestimated initially. The potential complication of soft tissue injury was not considered. Rhabdomyolysis was missed, and was not considered as one of the differential diagnoses in the management of patient with motor vehicle accident or prolonged muscle pain and lower limb weakness. This had resulted in bad prognosis and mortality in rhabdomyolysis. Early diagnosis and prompt treatment are highly recommended in such cases to save people's lives, give good prognosis, and give good results in future treatment and care of patients.

\section{CONCLUSION}

Soft tissue injury with large haematoma, ecchymosis and haemorrhage may take longer to heal, and carries higher risks of complications. Soft tissue injury should not be taken lightly because poor prognosis may result from improper management and care of patients. A list of guidelines and useful investigations can give a clear guide to the management of such cases. Useful information may be obtained by taking good history and undertaking a thorough physical examination. All trauma cases, especially patients presented with unresolved myalgia, a differential diagnosis of rhabdomyolysis should be considered. Daily monitoring of complication of soft tissue injury is warranted in such cases and institution of treatment should be done early.

\section{ACKNOWLEDGEMENTS}

The authors thank the doctors and staff at the Universiti Kebangsaan Malaysia Medical Centre, and the officer from Police Diraja Malaysia their assistance in the investigation of the case. 


\section{REFERENCES}

1. Best TM. Soft-tissue injuries and muscle tears. Clin Sports Med 1997; 16(3): 419-34.

2. Alessandrino F, Balconi G. Complications of muscle injuries. J Ultrasound 2013; 16(4):21522.

3. G Efstratiadis, A Voulgaridou, D Nikiforou, A Kyventidis, E Kourkouni and $G$ Vergoulas. Rhabdomyolysis updated. Hippokratia. 2007 Jul -Sep; 11(3): 129-137.

4. Patrick A. Torres, John A. Helmstetter, Adam M. Kaye, Alan David Kaye. Rhabdomyolysis: Pathogenesis, Diagnosis, and Treatment. Ochsner J. 2015 Spring; 15(1): 58-69.

5. Khan FY. Rhabdomyolysis: a review of the literature. Neth J Med 2009; 67: 272.

6. Zager RA, Gamelin LM. Pathogenetic mechanisms in experimental hemoglobinuric acute renal failure. Am J Physiol 1989;256: 446 -55 .

7. Keltz E, Khan FY, Mann G. Rhabdomyolysis. The role of diagnostic and prognostic factors. Muscles, Ligaments \& Tendons J 2013; 3(4): 303-12.

8. Cooper PN, Tapson J, AR Morley. Immunoperoxidase techniques and histology in the diagnosis of rhabdomyolysis related acute renal failure. J Clin Pathol, 1992; 45: 825-7.

9. Gianfranco Cervellin, Ivan Comelli, Giuseppe Lippi. Rhabdomyolysis: historical background, clinical, diagnostic and therapeutic features. Clin Chem Lab Med 2010; 48: 749-56.

10. Thomas MAB, Ibels LS. Rhabdomyolysis and acute renal failure. N Engl J Med 1985;15: 6238. 
